\title{
The Irregularity Change of the Technical State's Elements of the Vehicle: form Submission
}

\author{
Alexander Novikov ${ }^{1}$, Sergey Grebennikov ${ }^{2 *}$, and Alexander Grebennikov ${ }^{2}$ \\ ${ }^{1}$ Orel State University, 95 Komsomolskaya str., Orel, 302026, Russia \\ 2 Yuri Gagarin State Technical University of Saratov, 77 Politekhnicheskaya str., Saratov, 410054, \\ Russia
}

\begin{abstract}
Analyzed the use of the term «irregularity changes of technical condition of element's vehicle» to certain stages of the operational cycle. Specific examples and quantitative assessment of irregularity changes the structural parameters for the elements, working independently, and interacting in the units or systems of the vehicle.
\end{abstract}

\section{Introduction}

Irregular change in the technical condition of elements the automobile transport vehicles (ATV) is objectively inevitable due to the inability of the initial and subsequent conditions of their functioning, which would lead to the same result in the future for all degrees of freedom (one of the conclusions of the second law of thermodynamics [9]). Therefore it is important to define scopes of the term "irregularity of change of technical condition of elements of the vehicle" to characteristic stages of an operational cycle.

\section{Material and methods}

Indeed, the vehicle as a complex technical system consisting of more than 20 thousand components, each of which operates in a wide range of load, speed and thermal modes, different physic-chemical environments and circuits (mechanical, electrical, hydraulic, pneumatic) to achieve identical reliability is extremely difficult, primarily for economic reasons. In this regard, the elements of the vehicle's inherent unevenness of changes of technical condition; many of the details of the resource are less than the regulated life vehicles, which in turn determines the feasibility of executing the current and capital repairs (CR) - replacement of worn parts with new or refurbished. In this context, the term «unevenness of changes of technical condition» is often used when talking about structural irregularity of operational reliability of elements of the vehicle.

Structural inequality condition is usually reflected as a percentage of failures of the units and systems ATV (Figure 1), or item most of the wear parts and assembly units in the basic items, such as polygon distribution of failures of the engine components (Figure 2), for the depreciation period or to the CR. Its indicators are generally an indication of the degree of

* Corresponding author: asg@sstu.ru 
perfection of designs of vehicles of the same class. Their main drawback - the complexity of the data; they do not allow assessment of the internal alignment of individual behavior individual units in the system groups, as hidden in aggregate statistics, their operational reliability and also presented in the form regardless of the time frame for the use of the vehicle.

\section{Theory}

The concept of the term «Irregular changes of technical condition» is not limited to the region of parametric estimates of the structural irregularity of the reliability of ATV, it is broader, more varied and widespread in many areas addressed in the technical operation, as a science.

For example, an important component in the structure of measures to improve the reliability of the elements of the ATV, the researchers attributed to the developments for reducing uneven changes of structural parameter (wear) on the surface of the moving parts in the mates. Macro-deflections from ideal geometric forms characteristic of many parts having a cylindrical surface.

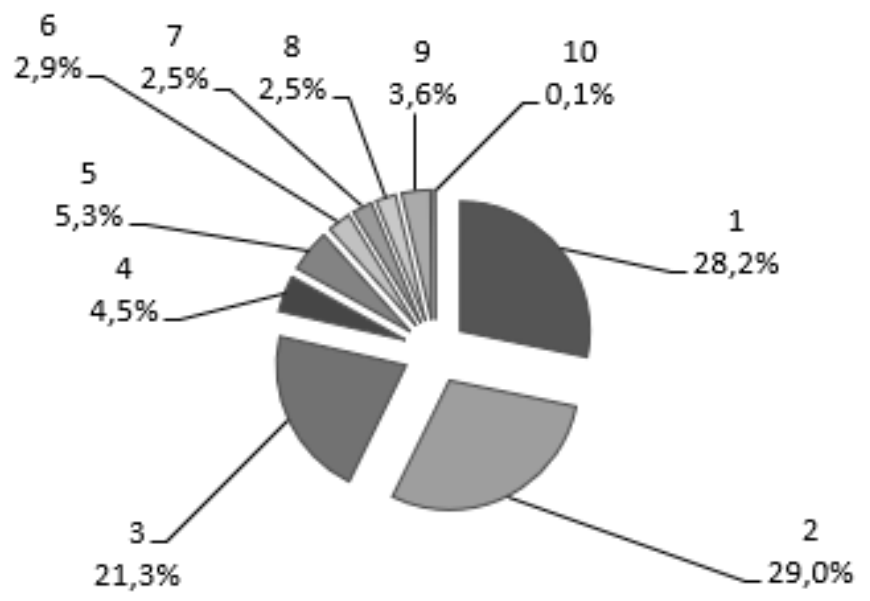

1 - wheels and tires; 2 - electric; 3 - the engine and its system; 4 - steering; 5 - brake system; 6 interior and platform superstructure; 7 powertrain; 8 - information support driver; 9 - the frame and suspension; 10 - air conditioning (refrigeration unit)

Fig. 1. The structural uniformity of distribution of the failures on the elements of the vehicles.

They are caused by the unequal distribution of dynamic loads on the contact surface in a longitudinally axial direction and in cross sections. Macro-deflections prevail in the form a taper and ovality, at least - convexity and celebratory. Quantification of taper (T), convexity, celebratory is the difference between the values of the structural parameters in the zones of maximum and minimum wear for the $i$-th in run-down belt longitudinal direction of

$$
T_{i}=S_{i \max }-S_{i \min }
$$

characteristics ovality is the difference between the values of mutually perpendicular diametrical wear in rundown of its cross section. 


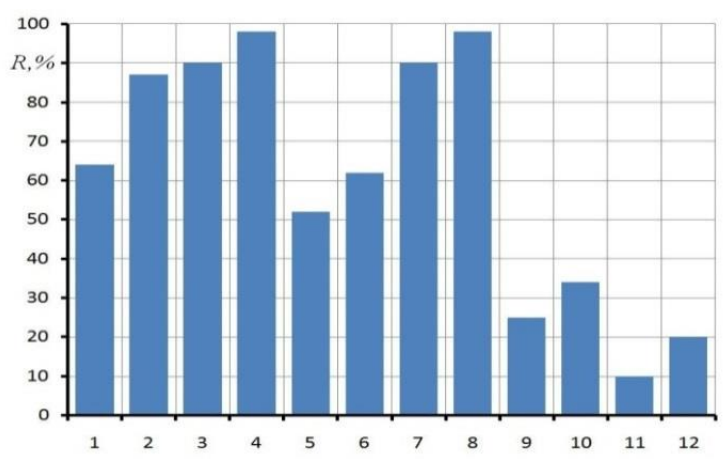

1 - cylinder liner; 2 - piston; 3 and 4 - oil and compression rings; 5 and 6 - main and connecting rod journals; 7 and 8 - indigenous and connecting rod liners; 9 - cams and necks of a camshaft; 10 - axles and roller tappets; 11,12 , respectively blocks and cylinder heads

Fig. 2. Diagram of the relative number of R engines YAMZ-236, -238, received the first $C R$ with extremely worn parts.

In addition to these forms of uneven wear of working surfaces of details for some mates characterized by more complex types of wear of the contact areas. Specialists of technical services of automobile transport enterprise (ATE) are well known typical plot of uneven wear of the cylinder liners, the crankshaft journals around the circumference and forming, camshaft lobes, the end surfaces of the gear teeth. The nature of their formation caused by variable loads from the action of the gas and inertial forces in the parts of the crank gear on the crankshaft angle of the internal combustion engine and transmission. Plots of uneven wear of the listed items show:

- the maximum wear of cylinder liners is in the stop position of the first compression ring when the piston is at TDC in the plane perpendicular to the axis of the crankshaft, caused by the action of lateral forces arising from the displacement of the piston in TDC;

- the crankshaft journals are out of round with a larger wear ear upper than the lower connecting rod bearings and, on the contrary, wear of the upper ear is less than the lower indigenous. The greatest wear on the rod journal is always formed on the side facing to the axis of the main journals, due to a unilateral action on the neck of inertia forces of the reciprocating moving masses and of centrifugal forces from the rotating parts of the connecting rod [2];

- in gearboxes and gearboxes from the leading bevel and cylindrical gears have excessive wear on the heads of teeth, and the driven legs of the teeth;

- from the camshaft area increased wear of the cam profile height. For all types of engines is characterized by a greater wear of cams operating with the exhaust valves, which are in a more intense load and thermal conditions than the intake valves.

Independently of the nature's formation of the irregular geometric shapes of parts, assessment of changes in structural parameters in such mating is carried out as in wear values in the most wear zones and sections.

More important and particularly important for the control system of the technical state of the ATV research aimed at revealing physical nature of tribological and other processes related to the description of functional dependencies of the transformation of technical conditions in different types of mates and interactive elements in the unit or the vehicle relative to its mileage. Similar information for units and systems ATV, operating in conditions of non-original, or received in the course of operation, the structural parameters, it is necessary to improve the methodology of diagnosis and predict the performance of the ATV. This interpretation of the objectives of the study the concept of «uneven changes of the technical state of elements of the vehicle» has multiple areas of application development, 
implementations that are specific to certain stages of the operational cycle of the vehicle, and characterizing different combinations of processes of interaction between mating parts. The authors of the article discusses the following three basic implementation of functional relationships summarizing and reflecting different sides of the uneven change of the technical condition of individual elements and the entire vehicle to specific periods of operation.

The essence of the first consists in disclosing negative transformation - degradation of technical condition of vehicle components in the process of its use, namely: non-linear dependence (dynamics) of increments of their structural parameters regarding race $l, S=f(l)$. As proved in [4] the parameters of the dynamics $S=f(l)$ a specific element of the vehicle lawfully can only be used in the run the mileage, when the structural connections in his mates, not broken regular maintenance impact. The technical condition of the element is determined by the measured structural change - the «point» of his finding on the dynamics in the area of health (Figure 3), limited source with a nominal value Nom obtained in the production process according to the technical documentation, and preteen nymphet., in which the operation of ATV is impossible - it is necessary to perform a maintenance service (SM) or repair with the aim of preventing failure.

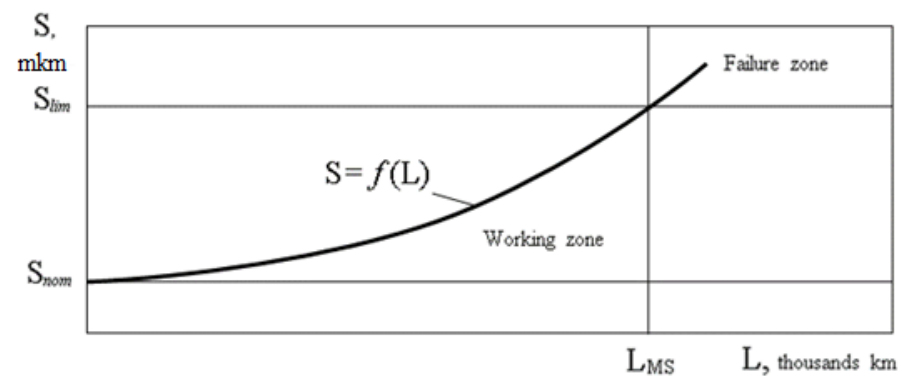

Fig. 3. The unevenness structural change of the $S$ parameter item ATV for is not $L$.

For the majority elements of the vehicle dynamics, the wear of the current run of 1 with sufficient accuracy described by an exponential dependence $[1,2,3]$

$$
S=S_{0} \mathrm{e}^{b l}
$$

where $S_{0}$ - is the value of the structural parameter after the running-in stage; $b$ - the coefficient of the intensification of wear, is determined by experimental data of a piecemeal measurement of wear.

At the same according to increase of non-uniformity of macro-deflections radial surfaces $\varepsilon_{H}$ from the ideal forms from the mileage of the vehicle, for example, taper and ovality of necks a cranked shaft

$$
\varepsilon_{H}=\varepsilon_{H 0} e^{b_{H \varepsilon} l},
$$

where $\varepsilon_{H 0}$-unevenness macro-deflections (1) the crankshaft journals after running.

Dependences $(2,3)$ indicate the progressive increase of the rate of wear and increasing the flow of failures as the development of online vehicle, and accordingly, the operating costs of their removal.

The purpose of the second analytical forms of representations of the unevenness change technical condition consists in taking account of the technical services of ATE consistent transformation of the indicators of trend lines speaker the structural parameters of the vehicle components in the overhaul cycle dependencies, shown graphically in Figure 4 [4].

On the chart (Figure 4) unevenness change of the technical condition of ATV, expressed via operational maintenance impacts in a consistent overhaul periods and life cycle, where 
the vertical solid lines allocated the costs of the capital repairs (CR), and the dashed turnaround of the vehicle. Analytical arguments [4], and many statistical material shows that the cost of restoration repairs from cycle to cycle increase $\mathrm{C}_{\mathrm{cr} 2}>\mathrm{C}_{\mathrm{cr} 1}$, and each subsequent post-repair operational period of the life cycle of ATV 1.3...2 times or more short than primary - primary, source. Has noted that after holding CR to the basic units of the vehicle, the cost of maintaining its efficiency in the early periods the next cycle operation are returned to the values close to the original, typical of new vehicles. However, due to the worst reliability indicators (technological level of recovered units after CR is much lower) cost increases is more «cool» non-linear dependence (2), with values of the ratio of the intensification of wear of $b(i+1)>b_{i}$.

Accounting irregularity overhaul cycles and speaker change condition significantly changes created through the planned maintenance system methodology justification of periodicities, the volume of technical impacts and material and labor costs during the overhaul, sufficiently long periods of operation of the exchange. This peculiarity should be taken into account in the justification of the recoverable resource system, because the deterioration of the speaker after a repair or CR leads to a decrease in the resource potential of the newly restored elements of the ATV $[3,4]$.

The third area of interpretation and presentation of non-uniformity of conditions relates to analytical descriptions of frictional processes in the most complex, kinematically interconnected parts with different structural parameters, together functioning as a part of the particular unit or system of the vehicle. These elements operate in the most responsible of defining technical and operational parameters for the ATV units (engines, injection pumps, gearboxes, transmission, and battery). Technical status of each item directly affects the wear rate of the other and the system as a whole $[1,2,3]$. This explains the fact that in the same units of vehicles of one model at the same time often there is a significant difference in the values of their wear and, consequently, resources. For example, we know: the durability of rolling bearings of the same size from the same batch of production, operating in strictly comparable conditions can differ up to 40 times [4].

In this regard, we note that at the present stage of development of preventive systems to ensure efficiency of ATV scientifically based methodology for decision-making on performance of repair work with guaranteed resource units associated with the selection of spare parts to replace extremely worn, which will operate as part of the restored system with different degree of wear of interrelated elements, no. But this problem arises daily in front of the technical services of ATE in the implementation of the artificial correction of the technical state of any element of the vehicle when the failure or warning.

Process adequate response technical services for a combination of changed structural parameters are functionally dependent elements of the vehicle, which was a failure, requires a specific approach to analyzing and deciding on the optimal formation of their dimensional chains, requiring, in some cases, replacement of one (out of service), and two or more parts. These tasks of frictional character were not given sufficient attention.

This is confirmed by a number of publications $[2,5,7,8,10,11,12,13]$ dedicated to the topic of the study. Most of them provides practical recommendations aimed at ensuring the uniformity of backlashes in coaxial friction pairs of the same name of the specific mechanisms of the ATV, and also draws attention to the difficulty of forming an opinion on the technical condition of the object according to the diagnostic results, made from the same elements with significant variation of the structural parameters. Researchers $[6,11]$ resource capabilities of engines in conditions of irregularity senses values in the same of their mates, said the feature: the higher the fluctuation of the clearance in the crankshaft bearings and the cylinder group, the less the degree of potential resource crank mechanism the main element of internal combustion engines. 


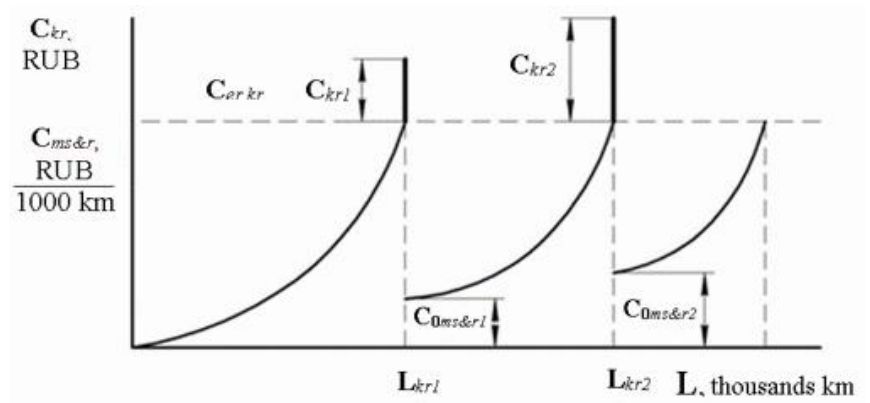

$C_{k r 1}, C_{k r 2}$ - value respectively the cost of the first and second CR; $C_{0 m s \& r l}, C_{0 m s \& r 2}$ respectively the cost values to and MS, given the initial periods of the following stages of operation of the exchange after CR

Fig. 4. Change specific operational $\mathrm{C}_{m s \& r}$ and repair costs of to ensure the operability of the elements of the ATV for is not L: $\mathrm{C}_{e r k r}$ - economically reasonable cost standard to assign the CR (on the run $\mathrm{L}_{\mathrm{kr} 1}, \mathrm{~L}_{\mathrm{kr} 2}$ ).

There is therefore, a problematic situation, namely, in the methodology justification of the capacity of the interrelated elements of ATV depending on the source or received in the course of operation indicators of the irregularity of their structural parameters. Work in this direction is conducted in the framework of fundamental research programs Saratov state technical University named of Gagarin Yu. A., some positive results of which are published $[3,4]$.

\section{Results}

The following are guidelines and evaluation criteria for the uneven change of the technical condition of the elements of the vehicle for the next stage of work on the analytical justification of the speaker on the types of mates and the nature of their interaction in the process of operation.

Still think citing studies that for each element pair of ATV under typical conditions of their work a distinctive, individual view of the dynamics of changes in technical condition that does not change over the entire period of operation. This postulate of invariability of the dynamics of the wear not without reason is one of the main original assumptions of the theory of durability of mechanical structures $[8,10,12]$. It based many of its applications, particularly the calculation and prediction of the resource, the regulation limit values of wear. However, these studies are not specified and consequently are not considered in the calculation methods, the presence of the speakers of the uneven change of the technical condition of objects of periodic irregularity that manifest in the form of significant deviations of the apparent «punctate» of experimental data from the trend lines speaker during various seasons of operation, and especially during the overhaul periods [4].

It is especially important to consider these circumstances when theoretical developments in the least explored region of the technical operation related to the justification of generalizing the assessment of the totality of conditions in related parts of mechanical systems ATV and purpose of the indicators of the limit of their States. The details of these systems can perform as a single kinematic function, such as torque transmission, and working autonomously with indirect kinematic connection through the housing body parts (shock absorbers, springs, ball joints, brake pads, discs, brake pads, etc.). The first of them work in direct relationship with each other, the second - in relative, but they wear out with different intensity, forming the irregularity of their current technical status in the objects of the ATV. 
Assessment of the degree of uniformity of the technical state of these elements is characterized by a maximum difference between the current structural and diagnostic parameters in an interrelated elements, is assigned to a certain mileage $l$ ATV

$$
\Delta \mathrm{S}_{\max (1)}=\mathrm{S}_{\max }-\mathrm{S}_{\min }
$$

where $S_{\max }, S_{\min }$, - respectively, the maximum and minimum values of the structural parameter (wear) in an interrelated mates.

For this reason, some of the eponymous elements of technical and regulatory requirements for the manufacture and operation of ATV manufacturers has limit restrictions on the irregularity index (4) describing technical condition when you are working them in collaboration. They caused a material adverse effect in excess of allowable irregularity in the working processes in internal combustion engines, handling, braking ability, tire wear, corrosion resistance of the body and other technical and operational characteristics of the ATV [3].

\section{Discussion}

The irregularity of the same name of elements functioning as a unit, assesses the relative value of the coefficient of uneven initial structural, operational and diagnostic parameters in the production process (at the stage of manufacture and assembly) or current (in service) and subsequent dynamics of its development on the predictable stages of operation of the exchange.

The use coefficients of two types, each of which has its own rational field of application and usage. Proposed by researchers at Zavolzhsky engine plant [6] ratio is more simple and is recommended for quantitative evaluation of the uneven wear of any set of the same name details

$$
E=\frac{S_{\max }}{\bar{S}}
$$

to determine the speaker of the uneven change of the technical condition of units and systems, which interact with the same parts and pairing, it is preferable to apply the ratio [4], which takes into account the extent of uneven wear mate

$$
\delta=\frac{S_{\max }-S_{\min }}{\bar{S}},
$$

where $\bar{S}$ - the average value of the analyzed wear eponymous items.

At this stage of the research it was found that for dynamically loaded bearings of the crankshaft are unevenly wear in the process of operation increases and is calculated by the formula [3].

$$
S_{H}=S_{H 0} e^{b_{H} l}
$$

where $S_{H_{0}}$-value source of non-uniformity of clearance in the bearings of the same name after the running-in stage; $b_{H}$ - coefficient of intensification of the uneven wear.

Given (7), it follows that the arithmetic mean of $S_{c p}$ for the same values of initial gaps in the corresponding bearings in the engine with a lower initial inequality $S_{H 0}$ gaps achievement of values of the marginal gap $S_{n}$, installed by the manufacturer of the internal combustion engine, is the most likely event. In addition, the resource of these bearings above. Considering the irregularity of the structural parameters the value of maximum permissible gap on projected mileage $l$ is calculated by the formula 


$$
S_{n \mu}=S_{n}-S_{c p}-0,5 S_{H 0} e^{b_{n} l}
$$

\section{Conclusion}

Consequently, the bearing life will decrease in proportion to the value of the initial unevenness $\mathrm{S}_{H O}$, and on the exponential dependence. It is therefore important to minimize the initial unevenness of the structural parameters of the same name mates and irregularity of parameters of working process in the cylinders of the internal combustion engine and transmission.

\section{References}

1. F. N. Avdonkin, Theoretical bases of maintenance vehicles./ F. N. Avdonkin. - M.: Transport, 215 p. (1985)

2. A. S. Denisov, Bases of formation of the maintenance cycle of the vehicle./ A. S. Denisov. - Saratov: Sarat. GOS. tehn. Univ, 352 p. (1999)

3. S. A. Grebennikov, Causation of the uneven change of the technical condition of your vehicle, S. A., Grebennikov, A. S. Grebennikov, A. S. Abeltsev. // Truck, No. 11.Page 3-11. (2016)

4. A.S. Grebennikov, Forecasting the Service Life of Elements with the Same Name in a Car as a Function of the Conditions of Their Interaction and Nonuniformity of Initial States / A.S. Grebennikov, S.A. Grebennikov, A.V. Konovalov, A.V. Kosareva // ISSN 1052-6188, Journal of Machinery Manufacture and Reliability, 2008, Vol. 37, No. 1, pp. 87-93. (C) Allerton Press, Inc., (2008)

5. R. Isermann, Fault-diagnosis systems: An introduction from fault detection to fault tolerance/ Isermann, R./ Springer Berlin Heidelberg, Pages 1-475. (2006)

6. A. D. Nazarov, Imbalances automotive engines: definition and valuation. / A. D. Nazarov. - M.: Mechanical Engineering. 272. (1992)

7. V.V. Nepomiluev, The study of possibilities of improving the quality of Assembly by individual selection of items / Nepomiluev V.V, E. A. Mayorov. // Assembly in mechanical engineering, instrument making. No. 10. - P. 43-46. (2006)

8. P. Pawlus, Change of cylinder surface topography in the initial stage of engine lifel Wear. Volume 209, Issue 1-2, August, Pages 69-83. (1997)

9. J. Prigogine, From being to becoming: time and complexity in the physical sciences/ J.Prigogine. - San Francisco: W. H. Freeman and Company, 327 p. (1980)

10. A.S. Pronikov, Parametric reliability of machines. / A.S. Pronikov.- M.: MGTU named N. E. Bauman, 560 p (2002)

11. V.A. Saninskyi, The group identified the assembly of the crankshaft of the slide with indigenous bearings and the crankshaft of internal combustion engines/ V. A.

Saninskyi// The mechanical engineering Bulletin. No. 4. - P. 31-36. (2006)

12. A.A. Shabana, Dynamics of multibody systems/ Shabana, A.A. /ISBN:9781107337213. Cambridge University Press, Pages 1-384. (2013)

13. A. A. Zvyagin, VAZ: Wear and repair / A. A. Zvyagin, M. A. Masina, A. M. Motin, V. B. Prokhorov; edited by A. A. Zvyagin. L. Polytechnic, 255 p. (1991) 